\title{
Birth Mode-Related Differences in Gut Microbiota Colonization and Immune System Development
}

\author{
M. Pilar Francino ${ }^{\mathrm{a}, \mathrm{b}}$ \\ a Unitat Mixta d'Investigació en Genòmica i Salut, Fundació per al Foment de la Investigació Sanitària i Biomèdica de \\ la Comunitat Valenciana (FISABIO-Salut Pública)/Institut de Biologia Integrativa de Sistemes (Universitat de València), \\ València, Spain; ${ }^{b}$ CIBER en Epidemiología y Salud Pública, Madrid, Spain
}

\author{
Keywords \\ C-section · Neonate · Gut microbiota - Immune system · \\ Tolerance · Allergy
}

\begin{abstract}
Background: The process of early gut colonization is extremely variable among individuals and is influenced by numerous factors. Among these, the mode of birth will strongly shape the early microbial exposure and immune environment of the neonate. Summary: Here, I review how the concomitant processes of microbiota and immune system development are altered by $\mathrm{C}$-section delivery and the effects of such alterations on long-term health. Key messages: C-section delivery impinges on microbiota and immune system development through various means: (i) if labor is lacking, intrauterine immune responses dependent on this process will not occur, affecting the immune environment of the neonate; (ii) the lack of exposure to the vaginal and fecal microbes of the mother will alter the type and diversity of the microbes that colonize the gut at birth; (iii) the different starting points in terms of microbial exposure and immune
\end{abstract}

environment will mark the course of microbiota and immune system development during the first months of life, generating multiple feedbacks between these 2 processes. Given that the first months of life represent a crucial time window in the ontogenesis of the immune system and the establishment of tolerance, $\mathrm{C}$-section delivery will impact on the lifelong risk of developing immune disease.

(c) 2018 S. Karger AG, Basel

\section{Immunological Effects of the Lack of Labor}

The type of birth is known to influence the immune environment of the neonate, as labor induces immune responses within the uterine cavity that will be absent in the case of elective C-sections [1]. Intra-uterine concentrations of inflammatory cytokines such as interleukin (IL) $-1 \beta$, IL-6, IL-8 and tumour necrosis factor- $\alpha$ (TNF- $\alpha$ ) are elevated during the normal course of term labor [2-4]. These cytokines play an important role during delivery, as they stimulate prostaglandin biosynthesis, thereby inducing myometrium contractility and ripening of the

\section{KARGER}

(c) 2018 S. Karger AG, Basel 
cervix [5]. The production of proinflammatory cytokines in the uterine environment is thought to be responsible for an activation of the fetal immune system during labor, reflected in an elevated number of total leukocytes in the neonate's circulation, mostly due to increased neutrophils, monocytes, and natural killer cells [6]. The stress of labor, however, has also been associated with decreased $\mathrm{T}$ lymphocytes and CD4+ helper T cells in cord blood [7, 8]. Moreover, labor produces a surge of cortisol, an immunosuppressive molecule that inhibits $\mathrm{T}$-cell effector function, leading to increased cortisol levels in umbilical cord blood from neonates delivered by spontaneous vaginal delivery [9].

Functional differences in cord blood mononucler cells dependent on delivery mode have also been reported. Steinborn et al. [10] detected a decreased production of the proinflammatory cytokine IL- 6 by monocytes recovered from cord blood after elective C-section. Ly et al. [11] observed that $\mathrm{C}$-section was associated with increased secretion by cord blood mononuclear cells of IL-13 and interferon- $\gamma($ IFN- $\gamma)$, cytokines that have been positively associated with atopy among children with a family history of this condition [12]. However, other studies have reported contradictory results regarding IFN- $\gamma$, including no differences associated to the delivery mode [1] and decreased levels in cord blood serum after elective C-sections [13]. In this last study, serum levels of various cytokines and cytokine receptors implicated in neonatal immunity were shown to be altered in C-section infants. In comparison to neonates delivered vaginally, those delivered by elective C-section had lower levels of IFN- $\gamma$, IL- 6 , IL- $1 \beta$, TNF- $\alpha$ and soluble TNF- $\alpha$ RI in umbilical cord blood, and lower levels of IL- $1 \beta$, soluble IL-2R, and TNF- $\alpha$ at day 1 of life, which persisted upto day 4 in the case of IL-2R and TNF- $\alpha$.

\section{Altered Exposure to Microbes at Birth and Different Gut Colonization Patterns}

In addition to the effects of the lack of labor-related immune responses, a C-section delivery will also greatly affect the exposure of the neonate to microbes at the moment of birth. These 2 effects will combine to impact on the development of the gut microbiota and on the ontogenesis of the immune system in infants born by C-section.

Numerous analyses have shown that birth by C-section alters the pattern of gut microbiota development relative to that of vaginally born infants. The very first mi- crobial inocula received by the newborn are very different in each case, as the infants born by C-section are not exposed to their mother's vaginal and fecal microbiota, and become initially colonized by skin bacteria, probably acquired from the surrounding environment [14]. Although most vaginal and skin bacteria do not seem to take hold in the infant gut, their presence may differentially affect the colonization abilities of other bacteria. Indeed, a lower gut microbiota diversity during the first week of life is reported for infants delivered by C-section [15]. Moreover, in surveys performed during the first week of life and at 1 month of age, infants born by $\mathrm{C}$-section are much less likely to be colonized by Bifidobacterium and Bacteroides at both times, whereas the presence of Clostridium difficile at 1 month is enhanced [16-19]. In addition, Huurre et al. [20] showed that infants born by Csection harbored lower amounts of total bacteria in their guts at 1 month of age, mainly due to a reduced number of bifidobacteria. Underrepresentation of Bacteroides is also frequently detected in the microbiota of $\mathrm{C}$-section infants surveyed at 3-4 months of age [16, 19, 21-23]. Longer term studies that have followed microbiota composition in infants during the first 2 years of life have confirmed an association of $\mathrm{C}$-section with delayed colonization of the Bacteroidetes phylum, and with lower total microbial diversity up to 2 years of age [22, 24]. Furthermore, differences between the microbiotas of C-section and vaginally born infants have been detected in analyses performed at 7 years of age [25]. However, other analyses have concluded that birth mode-related differences in the relative abundance of different gut microbiota taxa largely subside after the first year [24].

\section{Altered Ontogenesis of the Immune System}

Differences in microbial colonization patterns are important, not only because of their potential impact on the final composition of the microbiota, but also because they will influence the concomitant development of the infant's immune sytem. The maturation of the intestinal mucosa and its gut-associated lymphoid tissue is dependent on intestinal colonization. The gut-associated lymphoid tissue Peyer's patches, mesenteric lymph nodes and isolated lymphoid follicles require signals from the intestinal microbiota for complete development and/or recruitment of a mature complement of immune cells [26]. Furthermore, microbes shape the infant's incipient immune phenotype by engaging molecular receptors in a variety of gut immune cells and priming signalling cas- 
cades that direct cell differentiation and control the inflammatory status [27]. In this sense, experimental work in germ-free mice has shown that regulatory T cells (Treg) and invariant natural killer T cells can only be "educated" by microbial colonization during a specific postnatal time window to enable tolerance to later environmental exposures $[28,29]$. In other words, the repertoire of immune cells established at this critical period under the influence of the gut microbiota is going to affect the host's lifelong immune capacities and susceptibility towards disease [26, $30,31]$.

Therefore, the disrupted colonization due to C-section may be reflected in an altered development of the immune system, with potential long-term consequences. Several studies have addressed the impact of C-section on the development of $\mathrm{T}$ cell responses and the production of different cytokines. In the aforementioned study by Jakobsson et al. [22], the reduced diversity and delayed Bacteroidetes colonization associated with $\mathrm{C}$-section were accompanied by reduced Th1 responses during the first 2 years of life. Experimental work has shown that Bacteroides can exert strong effects on the immune system. In the case of $B$. fragilis, a surface polysaccharide is recognized by toll-like receptor 2 (TLR2) in Treg cells and induces production of IL-10 and other cytokines that affect the Th1/Th2 balance and promote immunotolerance [32]. Therefore, the absence of Bacteroides may be related to the reduction of Th1 responses detected in C-section infants.

Several other experimental studies in mice models have demonstrated associations between $\mathrm{C}$-section, microbiota development and immunity. Hansen et al. [33] showed that mice born by $\mathrm{C}$-section had a distinct bacterial profile at weaning and lower proportions of Foxp3+ regulatory $\mathrm{T}$ cells and tolerogenic $\mathrm{CD} 103+$ dendritic cells in mesenteric lymph nodes and spleens in adulthood, as well as a lower expression of the IL-10 gene. This demonstrates a long-term systemic effect of C-section birth on the regulatory immune system, even though no microbiota differences remained at adulthood. In the same direction, Lotz et al. [34] showed that C-section also affected the postnatal activation of intestinal epithelial cells (IECs), which needs to be critically regulated in order to maintain intestinal homeostasis. At birth, IECs are activated in response to the binding of bacterial lipopolysaccharide (LPS) molecules by TLR4, which results in intracellular cell signaling, transcriptional activation, and synthesis and secretion of chemokines. However, LPS susceptibility of IECs is only transient and is lost hours after birth, with no detectable proinflammatory effect, in- dicating perinatal induction of negative regulatory control mechanisms of epithelial TLR signaling. This phenomenon of transient postnatal epithelial activation is likely involved in the up-regulation of host defense molecules to prepare for subsequent microbial exposure, followed by a rapid acquisition of TLR tolerance to facilitate microbial colonization. This adaptive process, likely to be crucial to the establishment of gut homeostasis, was impaired in mice born by C-section, presumably due to low bacterial exposure. Human fetal IECs are known to be suceptible to LPS [35], so that postnatal epithelial activation might also occur in humans and be disrupted by the diminished bacterial diversity associated with delivery by C-section, potentially contributing to impaired tolerance acquisition and an increased risk of inflammatory conditions.

In accordance, humoral antibody-generating responses have also been found to vary between infants delivered vaginally or by C-section. Huurre et al. [20] showed that infants delivered by $\mathrm{C}$-section mount a stronger humoral immune response throughout the first year of life, with higher total numbers of immunoglobulin A (IgA)-, IgGand IgM-secreting $\mathrm{B}$ cells in peripheral blood. This might reflect decreased tolerance or an excessive antigen exposure that would be consistent with an increased tendency towards inflammation and the subsequent impairment of the gut barrier.

\section{Increased Incidence of Immune Disease}

As one would expect from the series of immune alterations that may follow from birth by $\mathrm{C}$-section, clinical evidence indicates that children born in this manner ar at higher risk for immune disorders, including type 1 diabetes and several types of allergies [36]. A large meta-analysis detected a $19 \%$ increase in type I diabetes in children born by $\mathrm{C}$-section after controlling for confounders such as gestational age, maternal age, and birth weight [37]. In the case of allergies, a meta-analysis of 26 studies demonstrated that C-section is associated with food allergy, atopy, allergic rhinitis, asthma, and hospitalization for asthma, but did not find evidence of an association with inhalant atopy and eczema/atopic dermatitis [38]. Further, a more recent systematic review involving 13 studies and 887,960 children confirmed that those delivered by C-section had increased risk of asthma up to the age of 12 [39]. Moreover, infants born after a repeat C-section without ruptured membranes have a $60 \%$ higher risk of asthma than infants born after an emergency C-section
Francino 
with ruptured membranes and/or prior labor, in accordance with their more altered immune environment and lower exposure to microbes at birth [40]. The increase in the risk of developing allergy after a C-section birth is particularly pronounced when there is a family history of the disease. In a Norwegian birth cohort, children of allergic mothers had a sevenfold increased risk of developing food allergy to egg, fish or nuts when born by C-section, but this was not the case in children whose mothers were not affected by the disease [41].

\section{Conclusion}

Birth by C-section has important effects on the intertwined processes of gut microbiota and immune system development. Furthermore, such effects can result in a variety of negative consequences for immune health, which are likely to last throughout life. Much further research is needed to understand all of the complex interactions between the early gut microbiota and the immune system and how they are affected by C-section delivery. Importantly, the impact of a C-section on immune development will be strongly dependent on whether it is an emergency or an elective procedure, as events such as membrane rupture and, especially, onset of labor will have important consequences in terms of immune response and microbial exposure. Elective $\mathrm{C}$-sections impose a greater alteration of the microbial and immune environment of the neonate and will likely have stronger health repercussions, as has already been seen for asthma risk.

\section{Disclosure Statement}

The author declares no conflicts of interest.

\section{References}

1 Thornton CA, Capristo CC, Power LL, et al: The effect of labor on neonatal T-cell phenotype and function. Pediatr Res 2003;54:120124.

2 Opsjln SL, Wathen NC, Tingulstad S, et al: Tumor necrosis factor, interleukin-1, and interleukin-6 in normal human pregnancy. Am J Obstet Gynecol 1993;169:397-404.

3 Steinborn A, Gunes H, Halberstadt E: Signal for term parturition is of trophoblast and therefore of fetal origin. Prostaglandins 1995; 50:237-252.

4 Steinborn A, Kuhnert M, Halberstadt E: Immunmodulating cytokines induce term and preterm parturition. J Perinat Med 1996;24: 381-390.

5 Romero R, Brody DT, Oyarzun E, et al: Infection and labor. III. Interleukin-1: a signal for the onset of parturition. Am J Obstet Gynecol 1989;160:1117-1123.

6 Thilaganathan B, Meher-Homji N, Nicolaides KH: Labor: an immunologically beneficial process for the neonate. Am J Obstet Gynecol 1994;171:1271-1272.

7 Pittard WB 3rd, Schleich DM, Geddes KM, Sorensen RU: Newborn lymphocyte subpopulations: the influence of labor. Am J Obstet Gynecol 1989;160:151-154.

8 Samelson R, Larkey DM, Amankwah KS, McConnachie P: Effect of labor on lymphocyte subsets in full-term neonates. Am J Reprod Immunol 1992;28:71-73.

9 Ruth V, Hallman M, Laatikainen T: Corticotropin-releasing hormone and cortisol in cord plasma in relation to gestational age, labor, and fetal distress. Am J Perinatol 1993; 10:115-118.
10 Steinborn A, Sohn C, Sayehli C, et al: Spontaneous labour at term is associated with fetal monocyte activation. Clin Exp Immunol 1999;117:147-152.

11 Ly NP, Ruiz-Perez B, Onderdonk AB, et al: Mode of delivery and cord blood cytokines: a birth cohort study. Clin Mol Allergy 2006; 4:13.

12 Rowe J, Heaton T, Kusel M, et al: High IFNgamma production by CD $8+\mathrm{T}$ cells and early sensitization among infants at high risk of atopy. J Allergy Clin Immunol 2004;113:710716.

13 Malamitsi-Puchner A, Protonotariou E, Boutsikou T, et al: The influence of the mode of delivery on circulating cytokine concentrations in the perinatal period. Early Hum Dev 2005;81:387-392.

14 Dominguez-Bello MG, Costello EK, Contreras $\mathrm{M}$, et al: Delivery mode shapes the acquisition and structure of the initial microbiota across multiple body habitats in newborns. Proc Natl Acad Sci U S A 2010;107:1197111975.

15 Rutayisire E, Huang K, Liu Y, Tao F: The mode of delivery affects the diversity and colonization pattern of the gut microbiota during the first year of infants' life: a systematic review. BMC Gastroenterol 2016;16:86

16 Gronlund MM, Lehtonen OP, Eerola E, Kero P: Fecal microflora in healthy infants born by different methods of delivery: permanent changes in intestinal flora after cesarean delivery. J Pediatr Gastroenterol Nutr 1999;28: 19-25.

17 Penders J, Thijs C, Vink C, et al: Factors influencing the composition of the intestinal microbiota in early infancy. Pediatrics 2006; 118:511-521.

18 Biasucci G, Benenati B, Morelli L: et al; Cesarean delivery may affect the early biodiversity of intestinal bacteria. J Nutr 2008; 138:1796S1800 S.

19 Hesla HM, Stenius F, Jaderlund L, et al: Impact of lifestyle on the gut microbiota of healthy infants and their mothers - the ALADDIN birth cohort. FEMS Microbiol Ecol 2014;90:791-801.

20 Huurre A, Kalliomaki M, Rautava S, et al: Mode of delivery - effects on gut microbiota and humoral immunity. Neonatology 2008; 93:236-240.

21 Azad MB, Konya T, Maughan H, et al: Gut microbiota of healthy Canadian infants: profiles by mode of delivery and infant diet at 4 months. CMAJ 2013;185:385-394.

22 Jakobsson HE, Abrahamsson TR, Jenmalm MC, et al: Decreased gut microbiota diversity, delayed Bacteroidetes colonisation and reduced Th1 responses in infants delivered by caesarean section. Gut 2014;63:559-566.

23 Kabeerdoss J, Ferdous S, Balamurugan R, et al: Development of the gut microbiota in southern Indian infants from birth to 6 months: a molecular analysis. J Nutr Sci 2013; 2:e18.

24 Bokulich NA, Chung J, Battaglia T, et al: Antibiotics, birth mode, and diet shape microbiome maturation during early life. Sci Transl Med 2016;8:343ra382.

25 Salminen S, Gibson GR, McCartney AL, Isolauri E: Influence of mode of delivery on gut microbiota composition in seven year old children. Gut 2004;53:1388-1389. 
26 Maynard CL, Elson CO, Hatton RD, Weaver CT: Reciprocal interactions of the intestinal microbiota and immune system. Nature 2012; 489:231-241.

27 Romagnani S: Regulation of the T cell response. Clin Exp Allergy 2006;36:13571366.

28 Hansen CH, Nielsen DS, Kverka M, et al: Patterns of early gut colonization shape future immune responses of the host. PLoS One 2012;7:e34043.

29 Olszak T, An D, Zeissig S, et al: Microbial exposure during early life has persistent effects on natural killer $\mathrm{T}$ cell function. Science 2012; 336:489-493.

30 Francino MP: Early development of the gut microbiota and immune health. Pathogens 2014;3:769-790.

31 Brugman S, Perdijk O, van Neerven RJ, Savelkoul HF: Mucosal immune development in early life: setting the stage. Arch Immunol Ther Exp (Warsz) 2015;63:251-268.
32 Round JL, Mazmanian SK: Inducible Foxp3+ regulatory T-cell development by a commensal bacterium of the intestinal microbiota. Proc Natl Acad Sci U S A 2010;107:1220412209.

33 Hansen CH, Andersen LS, Krych L, et al: Mode of delivery shapes gut colonization pattern and modulates regulatory immunity in mice. J Immunol 2014;193:1213-1222.

34 Lotz M, Gutle D, Walther S, et al: Postnatal acquisition of endotoxin tolerance in intestinal epithelial cells. J Exp Med 2006;203:973984.

35 Fusunyan RD, Nanthakumar NN, Baldeon ME, Walker WA: Evidence for an innate immune response in the immature human intestine: toll-like receptors on fetal enterocytes. Pediatr Res 2001;49:589-593.

36 Neu J, Rushing J: Cesarean versus vaginal delivery: long-term infant outcomes and the hygiene hypothesis. Clin Perinatol 2011;38:321331.
37 Cardwell CR, Stene LC, Joner G, et al: Caesarean section is associated with an increased risk of childhood-onset type 1 diabetes mellitus: a meta-analysis of observational studies. Diabetologia 2008;51:726-735.

38 Bager P, Wohlfahrt J, Westergaard T: Caesarean delivery and risk of atopy and allergic disease: meta-analyses. Clin Exp Allergy 2008; 38:634-642.

39 Keag OE, Norman JE, Stock SJ: Long-term risks and benefits associated with cesarean delivery for mother, baby, and subsequent pregnancies: systematic review and meta-analysis. PLoS Med 2018;15:e1002494.

40 Renz-Polster H, David MR, Buist AS, et al: Caesarean section delivery and the risk of allergic disorders in childhood. Clin Exp Allergy 2005;35:1466-1472.

41 Eggesbo M, Botten G, Stigum H, et al: Is delivery by cesarean section a risk factor for food allergy? J Allergy Clin Immunol 2003;112: 420-426. 